\title{
Primer congreso de la Sociedad Uruguaya de Nutrición (SUNUT) "Desde la evidencia científica a la práctica clínica", vía online, del 9 al 12 de noviembre de 2020
}

\author{
First congress of the Uruguayan Nutrition Society (SUNUT) \\ "From scientific evidence to clinical practice", Online, November 9-12, 2020 \\ Primeiro congresso da Sociedade Uruguaia de Nutrição (SUNUT) \\ "Das evidências científicas à prática clínica", Via Online, 9 a 12 de novembro de 2020
}

Marcelo Yaffé Krakauer

https://doi.org/10.35454/rncm.v4n1.240

Desde principio de este año, la Comisión Directiva de la Sociedad Uruguaya de Nutrición (SUNUT) ha puesto en marcha un proceso de modernización y reposicionamiento de esta Sociedad frente a las diferentes federaciones, sociedades, colegios y asociaciones tanto a nivel nacional como internacional, así como con las diferentes empresas e industrias vinculadas a la nutrición humana.

En los últimos meses y como forma de completar esta primera fase, se ha designado un Comité Organizador integrado por diferentes profesionales de nuestra Sociedad, con la finalidad de planificar y organizar el "Primer Congreso Internacional Virtual". Para lograrlo, y con el aval del Comité Científico, este Comité Organizador ha trabajado intensamente para poder ofrecer un evento de nivel internacional, con invitados de referencia tanto en lo académico como en lo científico, que abarcan las cinco disciplinas que integran nuestra Sociedad (Medicina de Adulto, Pediatría, Nutrición, Enfermería y Farmacia); esto de la mano de un programa científico variado y actualizado en los temas abordados, siempre uniendo la evidencia científica a la práctica clínica.

Como corolario de todo este trabajo mancomunado y gracias al apoyo de nuestros profesionales y la colaboración de la industria, entre el 9 y el 13 de noviembre del presente año, la SUNUT se realizó el "Primer Congreso Internacional Virtual”. Durante esos cinco días hemos tenido la posibilidad y el honor de escuchar a 30 expositores internacionales, quienes nos transmitieron sus conocimientos, junto a un panel de excelentísimos expertos nacionales, quienes nos brindaron sus experiencias, y los aportes del público, los cuales enriquecieron más cada una de las actividades para todos nosotros.

También es importante destacar que durante este Congreso y en forma simultánea, hemos tenido la participación de diferentes grupos de trabajo nacionales e internacionales, que han presentado diferentes proyectos científicos de investigación bajo la modalidad de posters, los cuales fueron meticulosamente evaluados por un Comité de notables internacionales.

Como "broche de oro" para el Congreso y para todo el trabajo realizado, para que este sea una gran instancia de integración y aprendizaje, y gracias a la generosidad de la Junta Directiva de la Asociación Colombiana de Nutrición Clínica, responsable de la Revista de Nutrición Clínica y Metabolismo, se nos honra con la posibilidad de compartir con ustedes una parte de nuestro Congreso a través de la publicación de los resúmenes de 11 de los 13 trabajos científicos presentados en este, incluido el trabajo ganador.

En esta breve reseña respecto a la planificación, organización y ejecución de nuestro Congreso queremos resaltar dos cosas que, a nuestro entender, fueron la clave del éxito: por un lado, el trabajo en equipo de manera 
multidisciplinaria con objetivos y metas comunes, pero con visiones diferentes del mismo problema y sus soluciones; $y$, por otro lado, el apoyo incondicional de todos los amigos, que de una u otra forma se hicieron presentes, lo que hizo que el evento haya sido doblemente exitoso.

Para concluir, quiero agradecer a la Comisión Directiva y a la Asamblea General de la SUNUT por la confianza depositada para encabezar la conducción de la Sociedad y este "Primer Congreso Internacional Virtual", junto a un gran grupo de excelentes y prestigiosos profesionales miembros de la SUNUT; a los presidentes y representantes de las diferentes asociaciones, colegios y sociedades "hermanas" que integran la FELANPE, así como a su Consejo Directivo por el apoyo incondicional. Especialmente, en esta oportunidad quiero agradecer a la honorable Junta Directiva de la Asociación Colombiana de Nutrición Clínica, que nos ha permitido publicar los resúmenes de los posters en su revista; a los distintos grupos de trabajo que apostaron al Congreso para presentar sus proyectos de investigación en la modalidad de posters; a la industria y las empresas nacionales, cuyos aportes nos ha permitido hacer frente a los gastos económicos que la organización del Congreso demandó; y, por último, pero no menos importante, a todos y cada uno de los asistentes, quienes a lo largo de los días estuvieron conectados y nos honraron con su presencia.

Sin más, me despido muy cordialmente y extiendo desde ya la invitación a nuestro "Segundo Congreso Internacional Virtual".

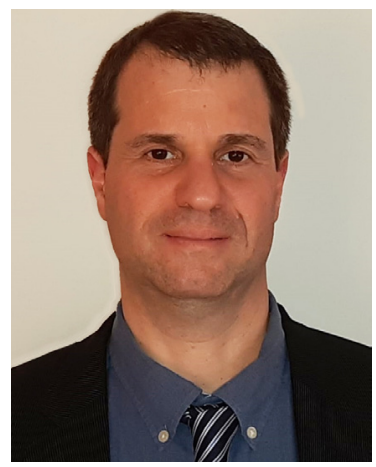

\section{Dr. Marcelo Yaffé Krakauer} Médico internista. Presidente de la SUNUT (2019-2021). Experto en Soporte Nutricional y Nutrición Clínica. Encargado de la Unidad de Soporte Nutricional Especial del Hospital Pasteur. Integrante de la directiva de la Sociedad Latinoamericana de Nutrición (SLAN). Miembro de la Federación Latinoamericana de Nutrición Clínica y Metabolismo (FELANPE)

Correo electrónico: marceloyaffe@movinet.com.uy

$1^{\text {er. }}$ Congreso Internacional Virtual de la Sociedad Uruguaya de Nutrición (SUNUT)
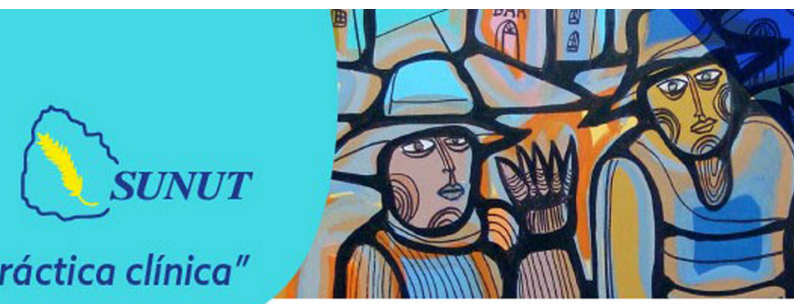


\title{
Primer congreso de la Sociedad Uruguaya de Nutrición (SUNUT) "Desde la evidencia científica a la práctica clínica", vía online, del 9 al 12 de noviembre de 2020
}

\author{
First congress of the Uruguayan Nutrition Society (SUNUT) \\ "From scientific evidence to clinical practice", Online, November 9-12, 2020 \\ Primeiro congresso da Sociedade Uruguaia de Nutrição (SUNUT) \\ "Das evidências científicas à prática clínica", Via Online, 9 a 12 de novembro de 2020
}

\begin{abstract}
ID: 1

\section{NIVEL DE CONOCIMIENTOS SOBRE ALIMENTACIÓN Y SU IMPACTO EN EL ESTADO NUTRICIONAL EN PACIENTES EN HEMODIÁLISIS}

Introducción: numerosos pacientes en hemodiálisis tienen un estado nutricional, ganancia de peso interdialítica y fosfatemia inadecuados. Es importante evaluar sus conocimientos y cómo influyen en los parámetros nutricionales y bioquímicos. La educación alimentaria nutricional se relaciona con una menor transgresión y una mayor adherencia al tratamiento nutricional.

Objetivos: determinar si existen diferencias significativas entre el estado nutricional, la ganancia de peso interdialítica y la fosfatemia entre los pacientes que tienen un conocimiento aceptable sobre alimentación, manejo de líquidos y del fósforo, que entre los que no.

Métodos: se incluyeron 42 pacientes en hemodiálisis en tratamiento durante más de 3 meses con asesoramiento nutricional. Diseño comparativo, a muestras independientes, prospectivo, observacional y transversal. La variable independiente fue nivel de conocimientos sobre alimentación y las variables

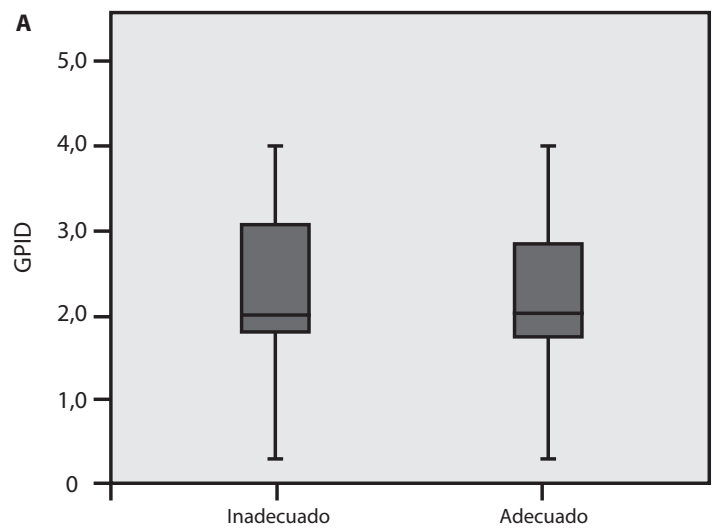

Nivel de conocimientos sobre alimentación dependientes fueron estado nutricional, ganancia de peso interdialítica y fosfatemia. Se utilizaron el Objective score of nutrition on dialysis (OSND), un cuestionario de elaboración propia y la historia clínica para la recolección de datos.

Resultados: el nivel de conocimientos fue adecuado en un $45 \%$. No se halló asociación estadísticamente significativa entre el nivel de conocimientos y el estado nutricional $(p=0,089)$, la ganancia de peso interdialítica $(p=0,507)$ y la fosfatemia ( $p=0,936)$ (Figura 1).

Conclusiones: no se halló asociación estadísticamente significativa entre las variables estudiadas. Sin embargo, hubo una tendencia a presentar un mejor estado nutricional en el grupo de pacientes con mayor nivel de conocimientos.

Palabras clave: conocimientos, hemodiálisis, estado nutricional.

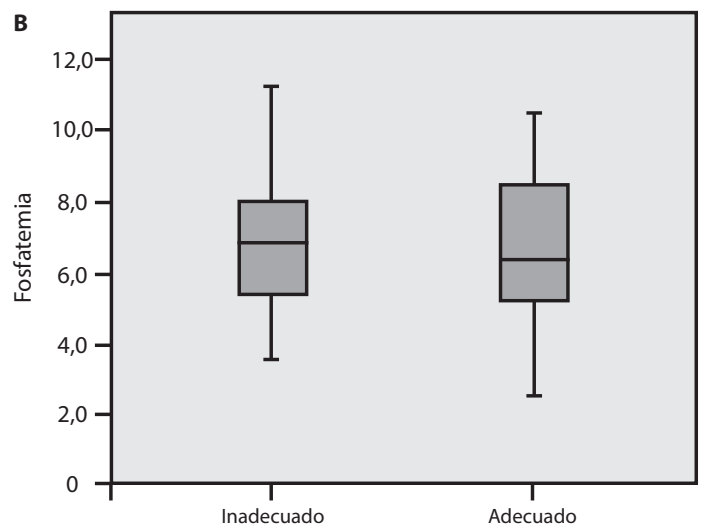

Nivel de conocimientos sobre alimentación

Figura 1. A. Comparación de medias de fosfatemia entre nivel de conocimiento adecuado versus inadecuado. B. Comparación de medias de ganancia de peso interdialítica entre nivel de conocimiento adecuado versus inadecuado. 
Abstract ID: 2

\section{EVALUACIÓN E INTERVENCIÓN NUTRICIONAL EN PACIENTES CON ESCLEROSIS LATERAL AMIOTRÓFICA ATENDIDOS EN UN CENTRO DE REFERENCIA DE MONTEVIDEO, URUGUAY}

La Rocca N.

E-mail: lic.larocca@gmail.com

País: Uruguay

Introducción: la esclerosis lateral amiotrófica (ELA) es una enfermedad neurológica degenerativa que afecta el funcionamiento de las motoneuronas, generando trastornos motores progresivos. Una adecuada nutrición es uno de los pilares del tratamiento, influyendo de forma positiva en la supervivencia y calidad de vida del paciente.

Objetivo: conocer las principales características de la evaluación e intervención nutricional, tomando como base experiencias con 12 pacientes.

Métodos: estudio experimental con una muestra de 12 pacientes diagnosticados con ELA, atendidos por un equipo multidisciplinario, que incluye consulta nutricional, en un Centro de Referencia para pacientes con ELA durante el período Mayo - Diciembre de 2015. Se estudió edad, sexo, presencia de otras patologías, estado nutricional, vías de alimentación y presencia de disfagia, y estrategias utilizadas para el tratamiento nutricional. Se valoró el estado nutricional de los pacientes mediante técnicas antropométricas (peso, talla, Índice de Masa Corporal, impresión clínica), se evaluó pérdida de peso en el tiempo, y se utilizó un modelo típico para evaluar la ingesta.

Resultados: se estudiaron 12 pacientes, 8 hombres y 4 mujeres, edad promedio 54 años. La mitad presentaron hipertensión y/o diabetes. La mayoría de los pacientes $(n=7)$ presentan normopeso, 4 presentan sobrepeso $u$ obesidad y sólo 1 bajo peso. La mayoría $(n=9)$ presentan riesgo nutricional por pérdida de peso significativa. La principal vía de alimentación es la oral, presentando disfagia en 8 pacientes. Las principales estrategias de intervención nutricional utilizadas fueron: modificación de consistencia de alimentos, alimentación hiperproteica y utilización de complementos nutricionales. Los pacientes que fueron controlados mostraron mejorías en cuanto a recuperación de peso y disfagia.

Conclusiones: los pacientes con ELA tienden a presentar riesgo nutricional y dificultades para deglutir, por lo que la nutrición es fundamental en el tratamiento.

Abstract ID: 3

\section{ESTRATEGIAS QUE MODIFICAN LAS CARACTERÍSTICAS SENSORIALES DE LAS VERDURAS PARA INCREMENTAR SU INGESTA}

López-Cardoso F, Gutiérrez-Arzápalo P, Cabrera-Chávez F, Nava-Delgado J, Alfonsina-Dezar G, Cárdenas-Torres F.

E-mail: ferfercardoso93@gmail.com

País: México

Introducción: una ingesta adecuada de verduras se ha relacionado con beneficios en la salud. Aunque la Organización Mundial de la Salud y la FAO recomienda consumir mínimo $240 \mathrm{~g}$ de verduras, esto está lejos de cumplirse. Esto se puede atribuir a diversas causas, como son la percepción del costo elevado de las verduras, el consumo de bebidas azucaradas y el sabor desagradable que se atribuye a las verduras. En este sentido, características sensoriales como el sabor y la textura son predictores del gusto por las verduras, por lo que el objetivo de la presente investigación es describir las estrategias implementadas para modificar los aspectos sensoriales de las verduras para incrementar el gusto y por lo tanto el consumo de estas.

Resultados: dentro de las estrategias encontradas, destacan los métodos de cocción, ya que las verduras cocidas en agua y al vapor reflejan una mejor aceptación, mostrando una mayor preferencia las cocciones de tiempo medio y largo (6-14 minutos). Por otra parte, existen estrategias donde se agregan condimentos, especias o aderezos para para modificar el sabor desagradable de las verduras e incrementar su aceptación y consumo. Otra estrategia que se basa en el sabor de las verduras es crear combinaciones entre éstas, donde se busca un aumento en el sabor dulce y la disminución del sabor amargo en la mezcla. La forma de servir las verduras, la cantidad de porción y el tamaño de las verduras son características que también influyen en la aceptación e ingesta.

Conclusión: dado la importancia del consumo de las verduras es importante encontrar estrategias que ayuden a incrementar su consumo, enfocadas principalmente a la modificación de las características sensoriales, ya que la característica del sabor es de mayor interés, por lo que sería conveniente evaluar el efecto de combinado de diversas estrategias para aumentar el impacto de las intervenciones.

Abstract ID: 4

\section{EVALUACIÓN DE LA ESTIMACIÓN DE PESO Y TALLA MEDIANTE ECUACIONES DE PREDICCIÓN EN ADULTOS MAYORES}

Banús PL, Romasco PJ, Scolaro LB.

E-mail: pau.banus@gmail.com

País: Argentina

Objetivo: estimar el grado de concordancia que existe entre las ecuaciones de predicción para estimar peso y talla y su valor real en la población adulta mayor.

Fundamentación: la toma de medidas antropométricas en los adultos mayores resulta una tarea ardua dado que estos pacientes tienen reducida su capacidad funcional, imposibilitando la bipedestación. Bajo estas circunstancias, diversos investigadores han elaborado fórmulas matemáticas para estimar el peso y la talla partiendo de la medición de distintos segmentos corporales. Sin embargo, actualmente existen pocos datos sobre la utilidad real de dichas ecuaciones.

Metodología: se incluyeron 118 adultos mayores internados o que asistieron a secciones especializadas en el cuidado del adulto mayor. Diseño comparativo a muestras relacionadas o intrasujeto, observacional, prospectivo y transversal. 
Resultados: los métodos de Rabito 2008 III, para el peso, y Chumlea 1985 y 1992, para la talla, fueron los que obtuvieron la media estimada más cercana a la media real (diferencia de $1,5 \mathrm{~kg}, 0,29 \mathrm{~cm}$ y $0,57 \mathrm{~cm}$, respectivamente), los que presentaron menor amplitud entre los límites de concordancia $(-7,8-11 \mathrm{~kg},-6,8-7,4$ cm y $-6,4-7,5 \mathrm{~cm}$, respectivamente) y un coeficiente de Lin correspondiente a un acuerdo moderado $(0,931,0,92$ y 0,928 , respectivamente) (Figura 1).
Conclusiones: podría considerarse la utilización de los métodos Rabito 2008 III y Chumlea 1985 y 1992 para la predicción del peso y talla, respectivamente, en las personas mayores postradas. En valores extremos, la estimación podría ser menos exacta.

Palabras clave: adulto mayor, peso, talla, ecuaciones, concordancia.

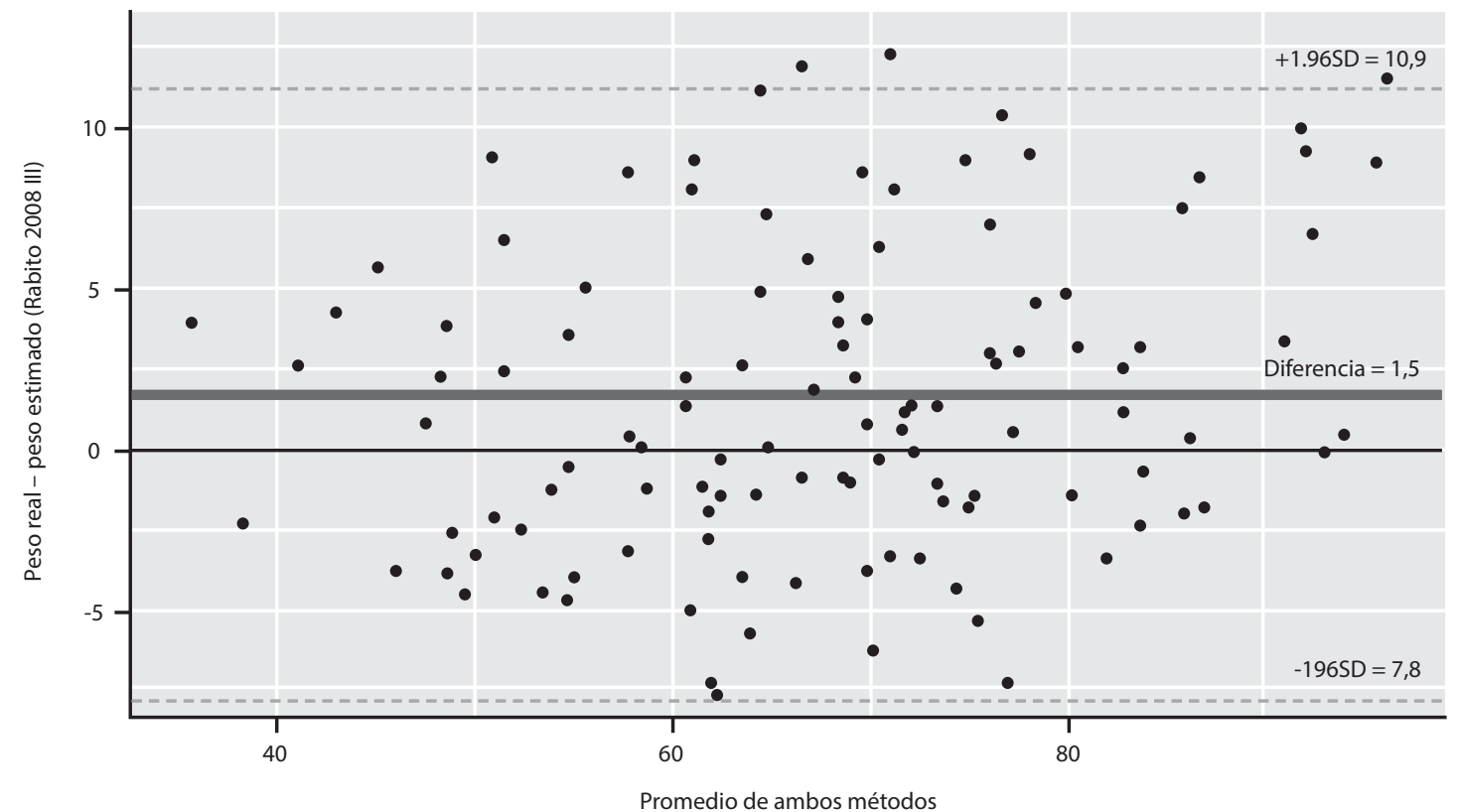

Figura 1. Bland-Altman peso real-estimado (Rabito 2008 III).

Abstract ID: 5

\section{ASOCIACIÓN ENTRE EL ESTADO NUTRICIONAL Y LA FRAGILIDAD EN ADULTOS MAYORES CON ENFERMEDAD CARDIOVASCULAR}

Introducción: la malnutrición en pacientes con enfermedad cardiovascular es altamente prevalente y aumenta la mortalidad. Además, la malnutrición está asociada a fragilidad en el envejecimiento. Sin embargo, se carece de estudios acerca de dicha asociación en adultos mayores que padezcan enfermedad cardiovascular.

Objetivo: examinar la posible asociación entre el estado nutricio y fragilidad en adultos mayores con enfermedad cardiovascular.

Método: estudio transversal analítico; 40 pacientes con enfermedad cardiovascular, $\geq 60$ años, quienes firmaron consentimiento informado. El estado nutricional fue evaluado mediante el Mini-Nutritional Assessment y de la fragilidad mediante el fenotipo de Fried. Los datos se presentan como mediana y frecuencias, las comparaciones se realizaron con la prueba $\mathrm{Ji}^{2}$ de Pearson.

Resultados: la edad fue 67 (63-71) años y $67 \%$ fueron hombres. Los resultados se muestran en la Tabla 1.

Conclusiones: este estudio sugiere que el estado nutricional normal se asocia a menor pre-fragilidad y mayor no fragilidad en adultos mayores con enfermedad cardiovascular.
Tabla 1. Estado nutricional según la fragilidad en adultos mayores con enfermedad cardiovascular

\begin{tabular}{|l|c|c|c|c|}
\hline & $\begin{array}{c}\text { Malnutrición } \\
\mathbf{n = 0}\end{array}$ & $\begin{array}{c}\text { Riesgo de } \\
\text { malnutrición } \\
\mathbf{n = 1 3}\end{array}$ & $\begin{array}{c}\text { Normal } \\
\mathbf{n = 2 7}\end{array}$ & $\mathbf{p}$ \\
\hline Frágil (\%) & $0(0,0)$ & $2(15,4)$ & $2(7,4)$ & \multirow{2}{*}{0,008} \\
\hline $\begin{array}{l}\text { Pre-frágil } \\
(\%)\end{array}$ & $0(0,0)$ & $10(76,9)$ & $9(33,3)$ & \\
\cline { 1 - 3 } $\begin{array}{l}\text { No frágil } \\
(\%)\end{array}$ & $0(0,0)$ & $9(33,3)$ & $16(59,3)$ & \\
\hline
\end{tabular}


Abstract ID: 6

\title{
FRECUENCIA DE CONSUMO DE BEBIDAS AZUCARADAS Y CAPACIDAD INTELECTUAL EN ADOLESCENTES
}

\author{
Ruvalcaba-Contreras N, García-Hernández O, Ramos-González N, Alfaro-Mendoza P.
}

E-mail: neriRC@hotmail.es

País: México

Introducción: un alto consumo de azúcar ha sido asociado a disminución de la concentración y la atención (componentes de la capacidad intelectual), pero existe un vacío de información sobre el consumo de bebidas azucaradas y la capacidad intelectual en adolescentes.

Objetivo: examinar la posible asociación del consumo de bebidas azucaradas y la capacidad intelectual en adolescentes.

Métodos: estudio transversal analítico realizado en 70 adolescentes aparentemente sanos de 15-19 años, quienes firmaron consentimiento informado. Los sujetos fueron cuestionados sobre su ingestión de bebidas azucaradas mediante un Cuestionario de Frecuencia de Consumo de Alimentos. La capacidad intelectual se evaluó mediante el Test de Matrices Progresivas de Raven y se clasificó como inferior al término medio (percentil $\leq 25$ ), término medio (percentil $>25$ y $<75$ ) y superior al término medio (percentil $\geq 76$ ). Los datos se presentan como promedio $\pm \mathrm{DE}$ y frecuencias (\%). Para las comparaciones se utilizó la prueba Ji-cuadrado.

Resultados: la edad media fue $17 \pm 0,89$ años, $53 \%$ fueron mujeres. Ver resultados en la Tabla 1.

Conclusiones: en esta muestra, los adolescentes cuya capacidad intelectual es inferior al término medio tienen un mayor consumo de soda.
Tabla 1. Niveles de azúcar en las bebidas

\begin{tabular}{|l|c|c|c|c|}
\hline & $\begin{array}{c}\text { Inferior al } \\
\text { término medio } \\
\mathbf{n = 1 2}\end{array}$ & $\begin{array}{c}\text { Término } \\
\text { medio } \\
\mathbf{n = 1 7}\end{array}$ & $\begin{array}{c}\text { Superior al } \\
\text { término medio } \\
\mathbf{n = 4 1}\end{array}$ & $\boldsymbol{p}$ \\
\hline $\begin{array}{l}\text { Bebida } \\
\text { azucarada casera } \\
\text { (ml/día) }\end{array}$ & $431 \pm 214$ & $490 \pm 143$ & $564 \pm 335$ & 0,388 \\
\hline Soda (ml/día) & $490 \pm 277$ & $280 \pm 120$ & $315 \pm 144$ & 0,047 \\
\hline $\begin{array}{l}\text { Jugo natural y } \\
\text { ultra procesado } \\
\text { (ml/día) }\end{array}$ & $227 \pm 114$ & $510 \pm 354$ & $431 \pm 217$ & 0,649 \\
\hline $\begin{array}{l}\text { Bebidas ultra } \\
\text { procesadas no } \\
\text { calóricas (ml/día) }\end{array}$ & $240 \pm 0$ & $340 \pm 137$ & $298 \pm 147$ & 0,688 \\
\hline
\end{tabular}

Abstract ID: 7

\section{ASOCIACIÓN ENTRE EL ESTADO NUTRICIONAL Y LA INFLAMACIÓN CON LA DEPRESIÓN Y LA IDEACIÓN SUICIDA EN LOS PACIENTES EN DIÁLISIS}

Ruvalcaba-Contreras N, Aguilar-Campos S, Valencia-Coronado R, Márquez-Herrera R, Calderón-Fabián A, Cortés-Sanabria L, Cueto-Manzano A, Rojas-Campos E.

E-mail: neriRC@hotmail.es

País: México

Introducción: el principal precursor de la ideación suicida es la depresión, que es el desorden psiquiátrico más común en pacientes en diálisis. Se ha reportado una asociación entre depresión y malnutrición e inflamación; pero no existe información sobre la posible relación entre malnutrición, inflamación e ideación suicida en pacientes con enfermedad renal crónica.

Objetivo: asociar el estado nutricional e inflamación con depresión e ideación suicida en pacientes en diálisis.

Metodología: estudio transversal analítico; 36 pacientes en diálisis $<18$ años quienes firmaron consentimiento informado. El estado nutricio se evaluó mediante el Dialysis Malnutrition Score y el inflamatorio mediante proteína C-reactiva y fibrinógeno. La depresión fue evaluada mediante el Inventario de Depresión de Beck y la ideación suicida mediante la Escala de Ideación suicida de Beck. Las comparaciones se realizaron con las pruebas U de Mann-Whitney y exacta de Fisher.

Resultados: ver Tabla 1.

Conclusiones: este estudio sugiere que no existe asociación entre el estado nutricional y depresión e ideación suicida en pacientes en diálisis.

Los pacientes con depresión y/o ideación suicida parecen presentar mayor inflamación.
Tabla 1. Estado nutricional e inflamatorio de acuerdo con depresión e ideación suicida en pacientes en diálisis

\begin{tabular}{|c|c|c|c|c|c|c|}
\hline & \multicolumn{3}{|c|}{ Depresión } & \multicolumn{3}{|c|}{ Ideación suicida } \\
\hline & $\begin{array}{c}\text { Sí } \\
(n=21)\end{array}$ & $\begin{array}{c}\text { No } \\
(n=15)\end{array}$ & $p$ & $\begin{array}{c}\text { Sí } \\
(n=23)\end{array}$ & $\begin{array}{c}\text { No } \\
(n=13)\end{array}$ & $p$ \\
\hline $\begin{array}{l}\text { Con } \\
\text { desnutrición } \\
\text { (\%) }\end{array}$ & 18 & 50 & 0,10 & 39 & 18 & 0,41 \\
\hline $\begin{array}{l}\text { Sin desnutrición } \\
(\%)\end{array}$ & 82 & 50 & & 61 & 82 & \\
\hline $\begin{array}{l}\text { Proteína } \\
\text { C-reactiva } \\
(\mathrm{mg} / \mathrm{L})\end{array}$ & $5,6(3-12)$ & $4,0(3-9)$ & 0,89 & $5,6(3-14)$ & $4,0(3-5)$ & 0,08 \\
\hline $\begin{array}{l}\text { Fibrinógeno } \\
\text { (mg/dL) }\end{array}$ & $\begin{array}{c}421(365- \\
555)\end{array}$ & $\begin{array}{c}365(252- \\
392)\end{array}$ & 0,02 & $\begin{array}{c}403(328- \\
532)\end{array}$ & $\begin{array}{c}375(278- \\
465)\end{array}$ & 0,26 \\
\hline
\end{tabular}


Abstract ID: 8

\section{MALNUTRICIÓN Y CAÍDAS EN ADULTOS MAYORES DE UNA COMUNIDAD RURAL MEXICANA}

Ruvalcaba-Contreras N, Jaime-Rangel M, Fuentez-Miranda A, Briseño-López A, Montiel-Valenzuela G, González-López L.

E-mail: neriRC@hotmail.es

País: México

Introducción: el envejecimiento es un proceso fisiológico que suele propiciar la pérdida involuntaria de peso, lo cual implica una disminución de la masa muscular esquelética y podría conducir a un aumento de caídas, que son una importante causa discapacidad y muerte en ancianos, pero son escasos los datos sobre su relación con el estado nutricional.

Objetivo: asociar el estado nutricional y las caídas en adultos mayores de una comunidad rural mexicana.

Metodología: estudio transversal analítico; 74 pacientes $>60$ años quienes firmaron consentimiento informado. El estado nutricional se evaluó mediante el Mini-Nutritional Assessment. Se preguntó a los sujetos; ¿cuántas caídas tuvo durante en el último año? Las comparaciones se realizaron con $\mathrm{Ji}^{2}$.

Resultados: la edad promedio fue 74 años y $64 \%$ fueron mujeres. Ver Tabla 1.

Conclusiones: este estudio sugiere que no existe asociación entre el estado nutricio y las caídas en adultos mayores de una comunidad rural mexicana.
Tabla 1. Estado nutricional según las caídas en adultos mayores de una comunidad rural mexicana

\begin{tabular}{|c|c|c|c|}
\hline & $\begin{array}{c}\text { 1 o más caídas } \\
(\mathbf{n = 4 2 )}\end{array}$ & $\begin{array}{c}\text { Sin caídas } \\
(\mathbf{n = 3 2 )}\end{array}$ & $\boldsymbol{p}$ \\
\hline $\begin{array}{l}\text { Estado nutricional (\%) } \\
\text { malnutrición }\end{array}$ & 10 & 16 & 0,15 \\
\hline $\begin{array}{c}\text { riesgo de } \\
\text { malnutrición }\end{array}$ & 69 & 47 & \\
\hline normal & 21 & 37 & \\
\hline
\end{tabular}

Abstract ID: 10

\section{FALLA INTESTINAL CRÓNICA EN PEDIATRÍA. UNIDAD DE NUTRICION ENTERAL Y PARENTERAL (UNEPP) CENTRO HOSPITALARIO PEREIRA ROSSEL}

Inverso A, Tanzi MN, Guisande A, Pietrafesa D.

E-mail: dampietrafesa81@gmail.com

País: Uruguay

Introducción: el fallo intestinal en pediatría se define como la reducción de la masa intestinal por debajo de las necesidades mínimas para mantener una adecuada digestión y absorción de nutrientes y fluidos requeridos para el crecimiento. La causa más frecuente es el síndrome de intestino corto. El tratamiento de estos niños se basa en el aporte de una nutrición parenteral total prolongada.

La existencia de equipos multidisciplinarios enfocados en el tratamiento integral de estos pacientes ha mejorado la supervivencia.

Objetivo: describir la evolución de pacientes pediátricos con fallo intestinal crónico, asistidos en la Unidad de Nutrición Enteral y Parenteral Pediátrica (UNEPP) del Centro Hospitalario Pereyra Rossell.

Métodos: en 10 años de la UNEPP se asistieron 4 pacientes con diagnóstico de fallo intestinal. Se registró sexo, edad de ingreso, causa del fallo intestinal, cirugías realizadas, longitud del intestino remanente, días de nutrición parenteral, complicaciones infecciosas, no infecciosas, evolución clínica, y crecimiento actual.

Resultados: cuatro pacientes, que ingresan en el primer trimestre de vida dos de sexo femenino y dos masculino. La etiología del fallo intestinal fue síndrome de intestino corto, tres gastrosquisis con atresia intestinal, uno onfalocele y atresia intestinal múltiple, asociada a inmunodeficiencia primaria. Todos requirieron hospitalización con alimentación parenteral prolongada y luego domiciliaria. Presentaron complicaciones infecciosas y no infecciosas. Ninguno presentó colestasis. En tres se realizaron técnicas de elongación intestinal. Un paciente falleció por causa de su inmunodeficiencia, dos están actualmente sin alimentación parenteral y uno la mantiene hasta el momento actual.

Conclusiones: el manejo integral del fallo intestinal crónico es complejo y requiere de un equipo multidisciplinario para su tratamiento y seguimiento a largo plazo.

Abstract ID: 11

\section{SARCOPENIA Y OSTEOSARCOPENIA COMO FACTORES DE RIESGO PARA DISCAPACIDAD FUNCIONAL EN ADULTOS MAYORES}

López-Teros M, Agudelo-Botero MT, Castro-Porras L, Sánchez-García S, Rosas Carrasco O.

E-mail: miriam.lopez@ibero.mx

País: México

Introducción: la discapacidad funcional (FD) es una condición geriátrica de alta prevalencia e impacto en la calidad de vida de los adultos mayores. Los cambios en la composición corporal durante el envejecimiento, así como la pérdida de la masa muscular y la masa ósea contribuyen a una menor capacidad funcional en los adultos mayores.
Objetivo: analizar la asociación entre sarcopenia (SP) y osteosarcopenia (OS) con la FD en adultos mexicanos de 50 años o más.

Métodos: un análisis transversal fue conducido con datos de 825 adultos de 50 años o más obtenidos de la cohorte FraDySMex (Frailty, Dynapenia and Sarcopenia in Mexican Adults). La composición corporal fue evaluada usando 
absorciometría dual de rayos X (DXA). El diagnóstico de sarcopenia se evaluó de acuerdo con los criterios del Consenso Europeo para la Definición y Diagnóstico de Sarcopenia (EWGSOP, 2019) y la osteopenia/osteoporosis fueron definidos de acuerdo a los criterios de la OMS (1995). La FD fue evaluada a través de las actividades básicas (ABVD) e instrumentales de la vida diaria (AIVD). Además, se estudiaron otras variables sociodemográficas, comorbilidad, estado cognitivo, síntomas depresivos y desempeño físico. Las asociaciones entre SP y OS con DF fueron evaluadas usando regresión logística múltiple.

Resultados: el promedio de edad fue de $70,3 \pm 10,8$ años y $77,1 \%$ fueron mujeres. Las prevalencias de FD, SP y la OS fueron $22,6 \%, 14,9 \%$ y $8,9 \%$, respectivamente. La SP fue asociada con alto riesgo de FD [OR: 1,70; IC 95\% 1,05, 2,76] y esta asociación fue mayor en OS [OR: 1,92; IC 95\% 1,11-3,33] (Tabla 1).

Conclusión: nuestros hallazgos sugieren que la presencia de SP y la OS incrementa el riesgo de FD en adultos de 50 años y más. Es importante desarrollar intervenciones oportunas para evitar la FD y con ello desenlaces adversos como fragilidad, institucionalización y mortalidad en los adultos mayores.

Palabras clave: sarcopenia, osteosarcopenia, discapacidad funcional, México, adultos mayores.
Tabla 1. Regresión logística entre sarcopenia y discapacidad funcional

\begin{tabular}{|c|c|c|}
\hline & RM[IC 95\%] & Valor $p$ \\
\hline \multicolumn{3}{|l|}{ Modelo 1} \\
\hline \multicolumn{3}{|c|}{ Sarcopenia } \\
\hline No & 1 & \\
\hline Sí & $1,70[1,05,2,76]$ & 0,03 \\
\hline \multicolumn{3}{|l|}{ Modelo 2} \\
\hline \multicolumn{3}{|c|}{ Osteosarcopenia } \\
\hline No & 1 & \\
\hline Sí & $1,92[1,11,3,33]$ & 0,019 \\
\hline
\end{tabular}

IC 95\%: intervalo de confianza; RM: razón de momios.

*Ambos modelos ajustados por edad, polifarmacia y malnutrición.

\section{HÁBITO DE DESAYUNO EN CENTROS DE ATENCIÓN AMBULATORIA HOSPITAL BRITÁNICO}

Amestoy A, Agulla L, Vanerio G.

E-mail: aleamestoy@gmail.com

País: Uruguay

Introducción: la omisión del desayuno es uno de los hábitos alimentarios de riesgo importante para el desarrollo de obesidad.

Objetivo: estudiar la prevalencia de la omisión del desayuno y factores de riesgo asociados.

Metodología: se realizó una encuesta a individuos que ingresaron a policlínicos del Hospital Británico de septiembre a noviembre de 2018 y 2019 . Se tomaron medidas de peso, talla y presión arterial.

Los datos se procesaron con software IBM SPSS ${ }^{\circledR}$ Versión 23. Se utilizaron medidas de tendencia central (media y desviaciones estándar) para variables continuas que distribuyen normal y porcentajes para variables categóricas. La comparación entre grupos para variables categóricas se realizó con chi-cuadrado.

Resultados: 791 encuestados, de 18 a 95 años con media de 56 (+/- 16,72) años, $75,5 \%$ mujeres y $24,5 \%$ hombres. $44,6 \%$ con sobrepeso y $19,4 \%$ obe- sidad ambos mayores en el grupo de los hombres ( $p<0,001) .45,2 \%$ de la población presentó al menos 1 factor de riesgo cardiovascular, destacándose hipertensión arterial medicada (52,91\%). El 94,6\% de los encuestados desayuna, hubo un aumento en los que desayunan de 2018 a $2019(p<0,05)$. Hay más obesidad en el grupo de los que no desayunan $(p<0,05)$. El $17,9 \%$ ha modificado los hábitos de desayuno, la mayoría por hábitos saludables (52\%). El $60,9 \%$ realiza actividad física, ésta es mayor en el grupo de los que desayunan $(p<0,05)$. 92,2 \% de los encuestados no fuma. No se encontró relación entre hábito de fumar y hábito de desayuno (Figura 1).

Conclusiones: encontramos mayor prevalencia de obesidad, menor consumo de frutas y menor actividad física en el grupo de los que no desayunan. Existen más hábitos de riesgo en la población que no desayuna.

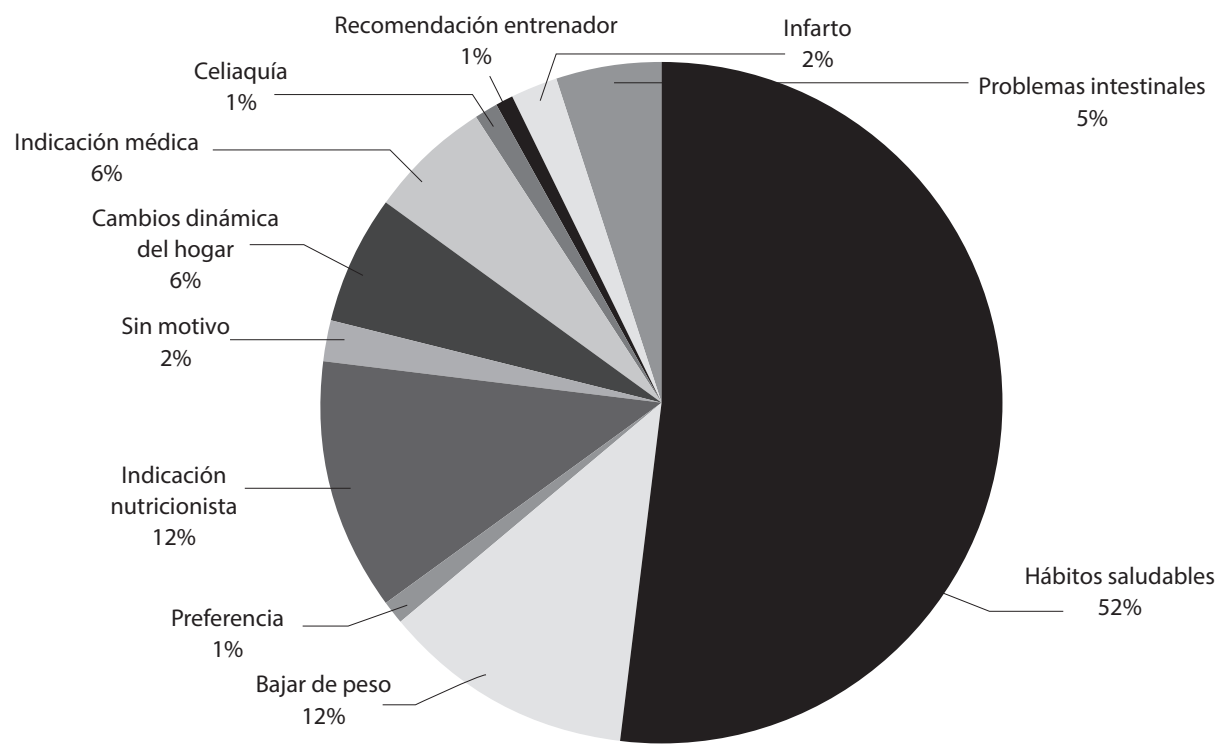

Figura 1. Motivos para modificar desayunos. 